\title{
Scirrhous Breast Carcinoma
}

National Cancer Institute

\section{Source}

National Cancer Institute. Scirrhous Breast Carcinoma. NCI Thesaurus. Code C7362.

An infiltrating ductal breast carcinoma associated with stromal fibrosis. 